\title{
IMPLEMENTATION OF CHORD LENGTH SAMPLING FOR TRANSPORT THROUGH A BINARY STOCHASTIC MIXTURE
}

\author{
T.J. Donovan and T.M. Sutton \\ Lockheed Martin Corp. \\ Schenectady, NY \\ Y. Danon \\ Mechanical, Aerospace, and Nuclear Engineering Department \\ Rensselaer Polytechnic Institute \\ Troy, NY \\ danon@rpi.edu
}

\begin{abstract}
Neutron transport through a special case stochastic mixture is examined, in which spheres of constant radius are uniformly mixed in a matrix material. A Monte Carlo algorithm previously proposed and examined in 2-D has been implemented in a test version of MCNP. The Limited Chord Length Sampling (LCLS) technique provides a means for modeling a binary stochastic mixture as a cell in MCNP. When inside a matrix cell, LCLS uses chord-length sampling to sample the distance to the next stochastic sphere. After a surface crossing into a stochastic sphere, transport is treated explicitly until the particle exits or is killed. Results were computed for a simple model with two different fixed neutron source distributions and three sets of material number densities. Stochastic spheres were modeled as black absorbers and varying degrees of scattering were introduced in the matrix material. Tallies were computed using the LCLS capability and by averaging results obtained from multiple realizations of the random geometry. Results were compared for accuracy and figures of merit were compared to indicate the efficiency gain of the LCLS method over the benchmark method. Results show that LCLS provides very good accuracy if the scattering optical thickness of the matrix is small $(\leq 1)$. Comparisons of figures of merit show an advantage to LCLS varying between factors of 141 and 5. LCLS efficiency and accuracy relative to the benchmark both decrease as scattering is increased in the matrix.
\end{abstract}

Key Words: stochastic mixture, transport, Monte Carlo, MCNP

\section{INTRODUCTION}

A binary stochastic mixture is defined as a random arrangement of two immiscible materials. Zimmerman and Adams (1) proposed the use of chord length sampling in Monte Carlo to simulate particle transport through such a mixture. Donovan and Danon (2) examined chord length sampling algorithms in 2-D for the special case of disks of constant radius mixed in a matrix material and proposed a Limited Chord Length Sampling (LCLS) algorithm that samples chords in the matrix material and treats transport through the spheres explicitly. This work extends the special case into a 3-D problem of spheres of constant radius mixed in a matrix material. 
Incorporation of a stochastic material treatment such as LCLS into a production Monte Carlo code such as MCNP (3) could find a wide audience. Applications include pebble bed reactor analyses, or heterogeneous shield or target designs. Also, the explicit treatment of the stochastic sphere allows for the inclusion of details such as multiple shells of varying material, such as is found in the fuel particle of the MIT High Temperature Gas Pebble Bed Reactor concept (4).

This work modified MCNP to incorporate the LCLS algorithm for fixed source problems. Test models are analyzed using LCLS as well as a benchmark method. Tally results are compared and the figure of merit (FOM) ratio for both methods is calculated.

\section{DESCRIPTION OF WORK}

\subsection{LCLS Algorithm}

The LCLS algorithm combines chord length sampling with limited explicit geometric representation of the stochastic mixture. The algorithm takes advantage of the closed, simple geometry of the stochastic spheres and treats these regions explicitly. When determining the next event, chord length sampling is used to sample the distance to a stochastic interface when a particle is within the matrix (material M1). Chord length sampling is used again to determine the position of the stochastic sphere. If the distance to the stochastic interface is less than the distance to collision and the distance to the nearest real surface, then the particle is moved forward to the surface of the stochastic sphere. Particle transport through the sphere is then carried out in the normal fashion.

\subsubsection{Matrix Chord Length Distribution}

Homogeneous mixing of the stochastic spheres in the matrix region is assumed. Excluding boundary effects, Torquato (5), showed that the chord length $\left(\lambda_{1}\right)$ distribution between spheres for such a mixture can be represented by the exponential form

$$
p\left(\lambda_{1}\right)=\frac{1}{\bar{\lambda}_{1}} \cdot \exp \left(-\frac{\lambda_{1}}{\bar{\lambda}_{1}}\right)
$$

where $\bar{\lambda}_{1}$ is the average chord length in the matrix material. Although the mixture boundaries may produce edge effects that influence how the stochastic spheres may be distributed, the LCLS method assumes that equation 1 still adequately represents the chord length distribution.

Although some efforts have been made to produce analytical approximations for $\bar{\lambda}_{1}(5,6)$, these approximations are not considered accurate enough to adequately test the LCLS method. Therefore $\bar{\lambda}_{1}$ is determined empirically for a given mixture geometry using a Monte Carlo technique. Details on how $\bar{\lambda}_{1}$ is determined are given in the description of the model, Section 2.4 .

The probability distribution of equation 1 is used to sample the distance to a stochastic interface, $D_{\text {I }}$. If $D_{\mathrm{I}}$ is less than the distance to the nearest real boundary crossing, $D_{\mathrm{B}}$, then additional work 
is performed to sample a stochastic sphere and test whether it can fit without overlapping the matrix cell boundaries.

\subsubsection{Stochastic Sphere Chord Length Distribution}

The stochastic sphere coordinates are sampled by first sampling a chord $\left(\lambda_{0}\right)$ through the sphere using the chord length distribution for a sphere (6)

$$
p\left(\lambda_{0}\right)=\frac{\lambda_{0}}{2 \cdot R^{2}},
$$

where $\mathrm{R}$ is the sphere radius. Figure 1 illustrates the $\lambda_{0}$ chord and its relationship to the stochastic sphere coordinates.

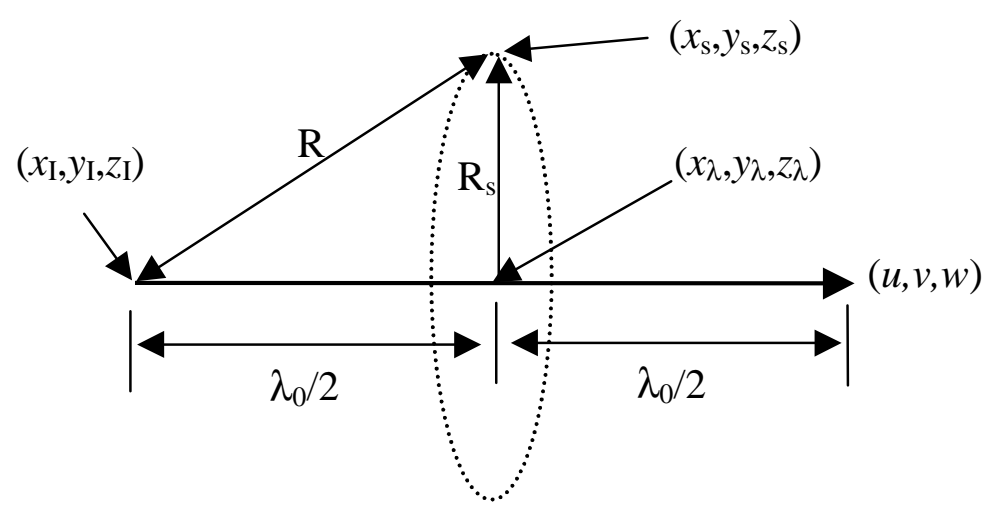

Figure 1: Sampling Stochastic Sphere Coordinates.

In Figure 1, $\left(x_{\mathrm{I}}, y_{\mathrm{I}}, z_{\mathrm{I}}\right)$ are the coordinates of the point where the particle will cross from the matrix material into the stochastic sphere, and $\left(x_{\lambda}, y_{\lambda}, z_{\lambda}\right)$ are the coordinates of the $\lambda_{0}$ chord midpoint. The chord midpoint coordinates are found by moving from point $\left(x_{\mathrm{I}}, y_{\mathrm{I}}, z_{\mathrm{I}}\right)$ a distance $\lambda_{0} / 2$ in the direction of particle motion described by the direction cosines $(u, v, w)$. The center of the stochastic sphere is $\left(x_{\mathrm{s}}, y_{\mathrm{s}}, z_{\mathrm{s}}\right)$, which lies on a circle in space described by

$$
\begin{gathered}
\left(x_{\lambda}-x_{s}\right)^{2}+\left(y_{\lambda}-y_{s}\right)^{2}+\left(z_{\lambda}-y_{s}\right)^{2}=R_{s}^{2}, \\
R_{s}^{2}=R^{2}-\left(\frac{\lambda_{0}}{2}\right)^{2} .
\end{gathered}
$$

The circle of equation 3 lies on the $x^{\prime} y^{\prime}$ plane of a newly defined coordinate system $\left(x^{\prime}, y^{\prime}, z^{\prime}\right)$, where the $z^{\prime}$ axis is in the direction of particle flight. A coordinate set is chosen from the circle by sampling an angle, $\theta$, from $(0,2 \pi)$ in the $x^{\prime} y^{\prime}$ plane. This angle defines a direction vector $\left(u_{s}^{\prime}, v_{s}^{\prime}, w_{s}^{\prime}\right)$ where $u_{s}^{\prime}=\cos (\theta), v_{s}^{\prime}=\sin (\theta)$, and $w_{s}^{\prime}=0$. This direction vector points from the chord midpoint to the sampled coordinate point, and the distance $\mathrm{R}_{\mathrm{S}}$ separates the two points. By performing a coordinate transformation, the sampled direction vector leading to the sphere coordinates is transformed back to the $(x, y, z)$ coordinate frame according to 


$$
\left(\begin{array}{l}
u_{s} \\
v_{s} \\
w_{s}
\end{array}\right)=\left(\begin{array}{ccc}
\frac{v}{\sqrt{u^{2}+v^{2}}} & \frac{u \cdot w}{\sqrt{u^{2}+v^{2}}} & u \\
\frac{u}{\sqrt{u^{2}+v^{2}}} & \frac{v \cdot w}{\sqrt{u^{2}+v^{2}}} & v \\
0 & -\sqrt{u^{2}+v^{2}} & w
\end{array}\right) \cdot\left(\begin{array}{l}
u_{s}^{\prime} \\
v_{s}^{\prime} \\
w_{s}^{\prime}
\end{array}\right)
$$

The sampled sphere coordinates are then

$$
\left(\begin{array}{l}
x_{s} \\
y_{s} \\
z_{s}
\end{array}\right)=R_{s} \cdot\left(\begin{array}{l}
u_{s} \\
v_{s} \\
w_{s}
\end{array}\right)+\left(\begin{array}{l}
x_{\lambda} \\
y_{\lambda} \\
z_{\lambda}
\end{array}\right) .
$$

An additional test is required to check that the sampled stochastic sphere does not intersect any surfaces of the matrix cell. If an intersection does occur, then the sampled sphere coordinates are rejected and the sampled distance to interface $D_{\mathrm{I}}$ is reset to a 'huge' value. If a stochastic sphere does not intersect the matrix cell surfaces, then the sampled value of $D_{\mathrm{I}}$ is included in the comparison of next-event distances and the minimum distance determines the next event.

If $D_{\mathrm{I}}$ is the minimum next-event distance, then a surface crossing with a stochastic sphere takes place. The particle is moved to the intersection point $\left(x_{\mathrm{I}}, y_{\mathrm{I}}, z_{\mathrm{I}}\right)$, it's cell location is changed from the matrix cell to the stochastic sphere, and the transport continues.

\subsection{Incorporation of LCLS into MCNP}

\subsubsection{Modifications to MCNP}

MCNP version 4C2 was modified to incorporate the LCLS method as described in Section 2.1. The only subroutine that was modified was history, which controls particle transport. The subroutine was modified to provide the following capabilities:

- Calculate $D_{\mathrm{I}}$, the distance to stochastic interface

- Sample stochastic sphere coordinates $\left(\mathrm{x}_{\mathrm{s}}, \mathrm{y}_{\mathrm{s}}, \mathrm{z}_{\mathrm{s}}\right)$ and test for overlap of the stochastic sphere with surfaces of the matrix cell

- Include $D_{\mathrm{I}}$ in the test for minimum distance to determine next event

- Translate particle into and out of the stochastic sphere as necessary

In addition to the modifications made to history, three new subroutines were created. These subroutines are called by history to perform the overlap test, and translate the particle in and out of the stochastic sphere. 


\subsubsection{MCNP Input to Define a Stochastic Mixture Cell}

The matrix cell is defined in the normal way and is filled with the matrix material. A representative stochastic sphere is created in a void region of zero importance in the model. Creating the stochastic sphere in such a region specifies the geometry and materials of the stochastic sphere but places it in a region of the model that particles do not transport through. Particle transport through the stochastic sphere can only occur if LCLS information is included in the input deck. The stochastic sphere may have multiple shells (each its own cell) built with concentric spheres.

The MCNP idum and rdum input arrays were used to specify LCLS information. The following information is specified:

\begin{tabular}{|c|c|}
\hline idum(1) & Matrix cell number \\
\hline $\operatorname{idum}(2)$ & $\mathrm{i}$, the number of concentric spheres making up the stochastic sphere. \\
\hline $\operatorname{idum}(2+1$ to $2+i)$ & The cell numbers of the stochastic sphere \\
\hline $\operatorname{idum}(3+\mathrm{i})$ & The surface number of the outermost surface of the stochastic sphere \\
\hline $\operatorname{idum}(4+\mathrm{i})$ & The number of the cell on the positive sense of surface idum $(3+i)$ \\
\hline $\operatorname{rdum}(1)$ & The average chord length in the matrix material \\
\hline $\operatorname{rdum}(2)$ & The radius of the stochastic sphere \\
\hline $\operatorname{rdum}(3)$ & $\mathrm{X}$ coordinate of the representative stochastic sphere \\
\hline $\operatorname{dum}(4)$ & Y coordinate of the representative stochastic sphere \\
\hline$d u m(5)$ & $\mathrm{Z}$ coordinate of the representative stochastic sphere \\
\hline
\end{tabular}

The only other inputs required to specify the stochastic mixture cell are the volumes of the matrix cell and the stochastic cells. These volumes must be entered using the VOL card so those tallies that use the cell volumes will be correctly normalized. The volumes that MCNP calculates automatically for these cells are not the true volumes of the stochastic mixture regions. The true matrix cell volume is equal to the 'empty' matrix cell minus the volume of the stochastic spheres that in reality are dispersed within it. Similarly, because the single representative stochastic sphere represents all of the stochastic spheres in the matrix, its true volume is equal to the volume of the single representative sphere multiplied by the number of spheres that it represents. If the stochastic sphere is modeled as a number of concentric spherical shells, then the representative volume of each shell cell must be specified.

\subsection{Optimization of Benchmark FOM}

This section discusses how benchmark tally results were obtained for comparison to the LCLS results. The benchmark tally result is obtained by running NITER MCNP jobs, each with a different random realization of the stochastic mixture, NPS particles per job, and a different random number seed. The average of the set of $y_{i}$ results is the benchmark tally result $y$, or

$$
y=\frac{\sum_{i} y_{i}}{N I T E R}
$$


If the ensemble averaged tally result $y$ were the only quantity of interest, the combination of NITER and NPS would not be of much concern. Both values would be set high enough to ensure that the error estimate on the mean result would be acceptably low and that the random effects due to the different possible mixture realizations are captured. However, the second objective of this study was to examine the differences in the FOM for the LCLS and Benchmark tallies. This comparison is only significant if the benchmark tally FOM has been optimized.

It can be shown that the variance in the population of $y_{i}$ results may be estimated as the sum of the geometric variance and the pooled stochastic variance of each of the $y_{i}$ results,

$$
S_{y_{i}}^{2}=S_{g}^{2}+\frac{S_{p}^{2}}{N P S}
$$

where $S_{y_{i}}^{2}$ is the variance of the $y_{i}$ results and $S_{g}^{2}$ is the geometric variance. The geometric variance is the variance in the NITER results due solely to the random effects of the placement of the stochastic spheres within the matrix cell. $S_{p}^{2}$ is the pooled, or averaged, stochastic variance of each of the NITER $y_{i}$ results. The term $S_{p}^{2} / N P S$ is an estimate of the variance in the NITER results due to the stochastic nature of the solution method.

The variance on $y$ is then

$$
S_{y}^{2}=\frac{S_{y_{i}}^{2}}{N I T E R} .
$$

The time to compute the average benchmark tally is

$$
T=\operatorname{NITER}(C+D \cdot N P S),
$$

where $C$ is an overhead time per realization and $D$ is a time per history. The FOM is typically defined as the inverse of the product of the relative uncertainty squared and the time required to compute the tally.

$$
F O M=\frac{1}{\left(S_{y} / y\right)^{2} \cdot T}
$$

In a typical Monte Carlo calculation, a single job is run with NPS particles. Since the relative uncertainty decreases as the inverse of the square root of NPS and the time is proportional to $N P S$, the FOM is expected to be a constant (i.e., independent of $N P S$ ) for a given tally. However, for the case in question, where the tally is an average of NITER results each obtained with NPS particles, the FOM is not independent of NPS. Substituting equations 8, 9, and 10 into equation 11, the resulting FOM expression is 


$$
F O M=\frac{y^{2}}{\left(\frac{S_{g}^{2}}{N I T E R}+\frac{S_{p}^{2}}{N I T E R \cdot N P S}\right) \cdot \operatorname{NITER}(C+D \cdot N P S)}
$$

NITER drops from equation 12, leaving FOM as a function of the variance parameters and NPS. The value of NPS that produces the maximum FOM is determined by differentiating Equation 12 with respect to NPS, setting the resulting expression to zero, and solving for NPS. The optimum value of NPS is found to be

$$
N P S_{o p t}=\sqrt{\frac{C \cdot S_{p}^{2}}{D \cdot S_{g}^{2}}} .
$$

To compute a benchmark tally for a given model, the variance and timing parameters are estimated by running a series of test jobs with varying combinations of NITER and NPS. The benchmark tally is then calculated again using the optimum NPS, and NITER is chosen to achieve the desired relative error or total number of histories.

It is important to note that in order to determine the optimum NPS for a given benchmark tally, the benchmark tally must be computed to a high degree of accuracy. Since the tally (and its FOM) is known, it is somewhat unrealistic to recalculate the tally simply to ensure that the last calculation was the most efficient. However, this approach is the most conservative and allows the comparison of LCLS FOM to the most ideal benchmark FOM.

For the models to be examined, the optimum NPS was determined for a single tally, but three tallies were actually computed. Because the remaining tallies were not optimized for their FOM, the LCLS to benchmark FOM ratios are not given for these tallies.

\subsection{Stochastic Mixture Model}

\subsubsection{Model Description}

The model consists of a sphere of radius $25 \mathrm{~cm}$, containing 781 randomly arranged spheres of $1 \mathrm{~cm}$ radius. The number and size of the stochastic spheres was chosen to obtain a $5 \%$ volume fraction of spheres in the matrix region. The stochastic spheres do not possess a shell substructure for this model. Because spheres were placed in the allowable volume sequentially, this method of creating a realization of the random mixture is known as Random Sequential Addition, or RSA (5). Randomly placed spheres were not permitted to overlap one another or the surface of the matrix sphere. In addition, the stochastic spheres were not permitted to fall on the origin located at the center of the matrix sphere. This restriction allows a point source to be specified in the matrix cell at the origin for all benchmark realizations. This non-physical restriction on the mixing of the stochastic spheres for the benchmark model is necessary because the LCLS modifications to MCNP do not currently permit source particles to originate from the stochastic spheres. Starting a point source at the origin in the LCLS model results in source particles originating in the matrix material. Because the source particle is born in the matrix 
material at the origin, a stochastic sphere cannot exist at that location at the beginning of the particle history. However, if a source particle scatters back near the origin later on in its history, the LCLS method may then sample a stochastic sphere that overlaps the origin.

Figure 2 shows plots of a benchmark realization of this model and the LCLS model of the same system. Each plot is a planar view of the $x-y$ view at $z=0$. In the LCLS model, the sphere outside and to the right of the matrix sphere is the representative stochastic sphere. In the benchmark model, each stochastic sphere is a unique cell placed randomly in the matrix sphere. Two matrix cells are defined, representing the two hemispheres in the positive and negative $x$ directions. Each matrix cell is the intersection of space inside the matrix sphere, in the positive or negative $\mathrm{x}$ space, and outside the 781 stochastic spheres.
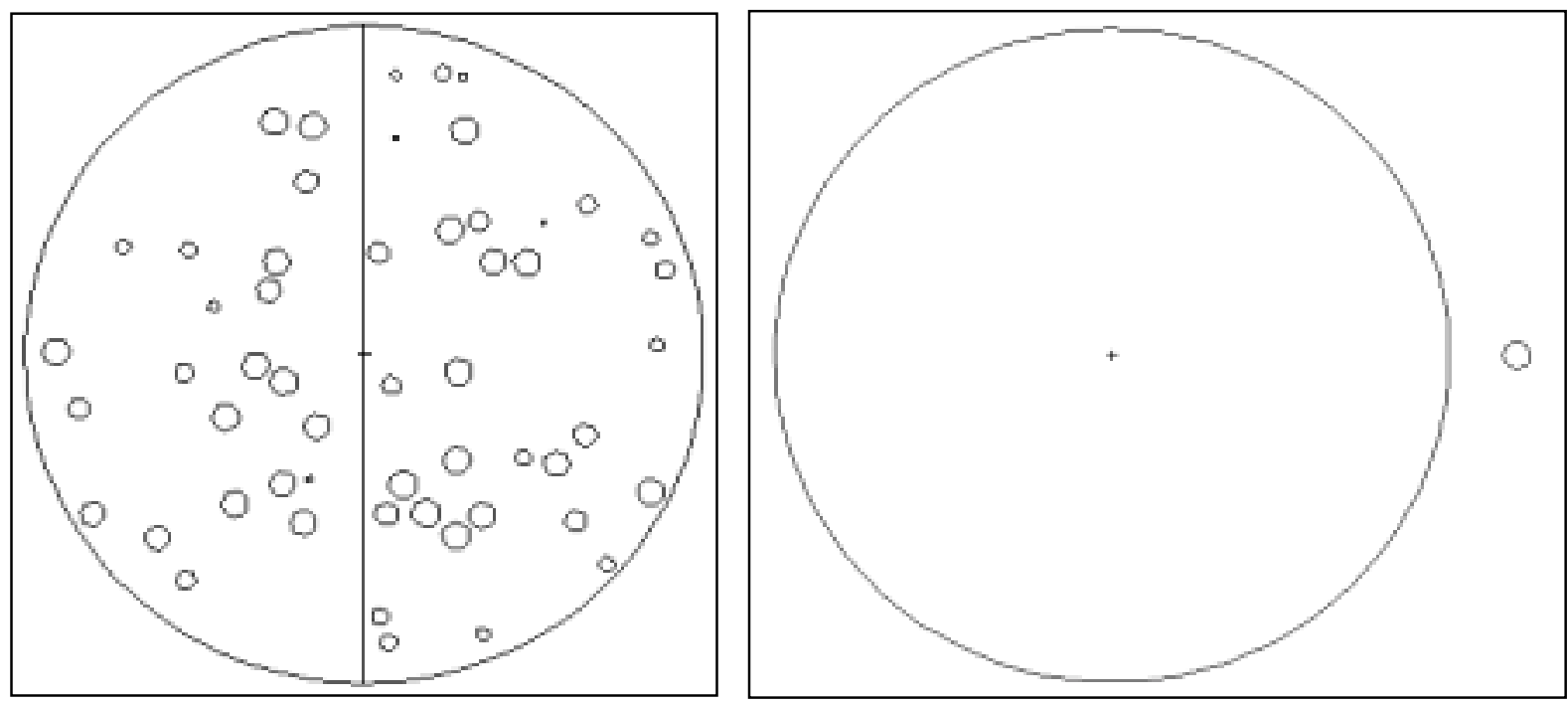

Figure 2: Benchmark MCNP model (left) and LCLS model (right). (x-y plane at z=0)

The matrix material (M1) is Nitrogen-14 and the stochastic sphere material (M0) is Boron-10. These materials were chosen for their smoothly varying cross sections in the thermal energy range. Also, the N-14 cross section is dominated by scattering whereas B-10 is a strong absorber. M0 density was specified to make the stochastic spheres black absorbers. Three different M1 densities were specified to study the effects of increasing scatter in the matrix. The three M1 densities were: $0.001 \mathrm{gm} / \mathrm{cc}, 0.1 \mathrm{gm} / \mathrm{cc}$, and $1 \mathrm{gm} / \mathrm{cc}$. For the thermal energy source, these densities correspond to mean free path lengths in the matrix of approximately $2000 \mathrm{~cm}, 20 \mathrm{~cm}$, and $2 \mathrm{~cm}$, respectively. Given that the average chord length in the matrix is approximately $20 \mathrm{~cm}$, the three matrix densities correspond roughly to optical thicknesses of 0.01 (transparent), 1 , and 10 (highly scattering), respectively.

The model was analyzed for a fixed thermal energy neutron source $(2.53 \mathrm{E}-8 \mathrm{MeV})$ in two different spatial distributions. The first distribution was a point source located at the center of the matrix sphere. The second distribution was uniformly spread throughout the matrix material. With two source distributions and three M1 densities, there were six cases analyzed in total. 
Three tallies were computed for each case. These tallies were: an F1 surface current tally on the surface of the matrix cell, an F4 cell flux tally in the matrix cell itself, and an F4 cell flux tally in the stochastic spheres. For the benchmark jobs the cell flux tally for the stochastic spheres is the average flux in all the explicitly modeled spheres.

\subsubsection{Determination of the Matrix Mean Chord Length}

The mean chord length in the matrix for this geometry was determined empirically to be 22.087 $\mathrm{cm}$. This value was determined by first modeling a realization of the stochastic mixture. The coordinate origin was defined as the center of the matrix sphere. The positive and negative $\mathrm{x}, \mathrm{y}$, and $\mathrm{z}$ axes (total of 6 lines) were then checked to determine how many did not have stochastic spheres overlapping them. This was repeated many times and the total number of instances where the 6 lines had no overlapping spheres was tallied. This value divided by six times the number of attempts defines the probability that a source particle originating at $(0,0,0)$ at an arbitrary direction would encounter no stochastic spheres along its initial trajectory. This probability, referred to as $p(0)$, is related to the average chord length $\bar{\lambda}_{1}$ in the matrix.

The relationship between $p(0)$ and $\bar{\lambda}_{1}$ can be expressed mathematically by starting a particle at $(0,0,0)$ and examining the method by which LCLS samples stochastic sphere locations. In order to simplify the integrals that will be developed, the following arguments assume that the stochastic sphere radius is much less than the radius of the matrix sphere.

From the starting location $(0,0,0)$, LCLS first samples a $\lambda_{l}$ chord in the matrix material. If $\lambda_{l}$ is greater than $R_{\mathrm{m}}-R$, where $R_{\mathrm{m}}$ is the matrix sphere radius and $R$ is the stochastic sphere radius, then the particle 'sees' no stochastic spheres along its flight path. This is a contribution to $p(0)$ but is not the only possibility. If $\lambda_{l}$ is between $\left(R_{m}-R\right)$ and $\left(R_{m}-2 R\right)$, there is a possibility that the sampled stochastic sphere overlaps the matrix sphere and will be rejected. If so, then particle again sees no stochastic sphere along its path. The total probability that a particle at the origin will see no stochastic spheres along its path is $p(0)$, and is equal to the sum of the two sampling probabilities described. This sum can be expressed formally using the two chord length distributions of equations 1 and 2, resulting in

$$
p(0)=\int_{R_{m}-R}^{\infty} \frac{1}{\bar{\lambda}_{1}} \cdot \exp \left(-\frac{x}{\bar{\lambda}_{1}}\right) d x+\int_{R_{m}-2 R}^{R_{m}-R}\left(\frac{1}{\bar{\lambda}_{1}} \cdot \exp \left(-\frac{x}{\bar{\lambda}_{1}}\right)\right) \cdot\left(\int_{2\left(R_{m}-R-x\right)}^{2 R} \frac{y}{2 \cdot R^{2}} d y\right) d x
$$

Equation 14 shows the relationship between $p(0)$ and the desired quantity $\bar{\lambda}_{1}$. Given the integration limits and the value obtained for $p(0)$, this expression was solved for $\bar{\lambda}_{1}$.

\section{RESULTS}

Tables I and II contain results for the point source and uniform matrix source, respectively. Each table contains the ensemble averaged benchmark results and the LCLS results for the three different matrix material densities. Each tally result also shows an estimate of the one standard 
deviation uncertainty on the last significant digit in parentheses. Figures of merit comparisons are only made for the F1 surface current tally on the matrix sphere.

For both source distributions, results for the first two matrix densities show excellent agreement (within 1\%) between the LCLS estimate and the benchmark ensemble averaged tally results. For the third matrix density case the accuracy of the LCLS method degrades significantly.

Increasing scattering in the matrix causes the LCLS results for leakage to underpredict the benchmark results. Logically, this also causes the LCLS results for the flux in the stochastic spheres to increase.

The FOM ratios on the surface current tally vary widely from a factor of 141 for the lowest matrix density point source case, to 4.8 for the medium matrix density uniform source case. Because of the overhead cost in creating random benchmark realizations, the LCLS model shows the most advantage over the benchmark method for models and tallies that possess the largest geometric variance (i.e., those benchmark models that require a larger NITER and smaller NPS for the optimized FOM). The point source case with the lowest matrix density produces very little scatter in the matrix. For particles to escape the matrix sphere they must have a free line of sight from the origin to the matrix surface along their direction of travel. The surface current tally for this case is more dependent on the random placement of the stochastic spheres than the other cases examined. As scattering increases in the matrix the geometric variance is reduced because a particle has an increased chance of channeling around stochastic spheres and contributing to the surface current tally.

Table I. Thermal neutron point source at $(0,0,0)$. Tally and FOM results for three sets of matrix material densities.

\begin{tabular}{|c|c|c|c|}
\hline \multicolumn{4}{|c|}{ Set1: $\rho 0=10[\mathrm{gm} / \mathrm{cc}], \rho 1=0.001[\mathrm{gm} / \mathrm{cc}]$} \\
\hline Tally & Benchmark & LCLS & $\frac{F O M_{L C L S}}{F O M_{\text {bench }}}$ \\
\hline Surface Current [n/source n] & $0.347(1)$ & $0.3459(5)$ & 141 \\
\hline Matrix Cell Flux [n/cm²/source n] & $2.420 \mathrm{E}-4(8)$ & 2.412E-4 (2) & - \\
\hline Stochastic Cell Flux [n/cm²/source n] & $8.62 \mathrm{E}-8(2)$ & $8.64 \mathrm{E}-8(1)$ & - \\
\hline \multicolumn{4}{|c|}{ Set 2: $\rho 0=10[\mathrm{gm} / \mathrm{cc}], \rho 1=0.1[\mathrm{gm} / \mathrm{cc}]$} \\
\hline Tally & Benchmark & LCLS & $\frac{F O M_{L C L S}}{F O M_{\text {bench }}}$ \\
\hline Surface Current [n/source n] & $0.2042(8)$ & $0.2034(4)$ & 87 \\
\hline Matrix Cell Flux [n/cm²/source n] & $2.472 \mathrm{E}-4(9)$ & 2.449E-4 (2) & - \\
\hline Stochastic Cell Flux [n/cm²/source n] & $9.05 \mathrm{E}-8(2)$ & 9.07E-8 (1) & - \\
\hline \multicolumn{4}{|c|}{ Set 3: $\rho 0=10[\mathrm{gm} / \mathrm{cc}], \rho 1=1[\mathrm{gm} / \mathrm{cc}]$} \\
\hline Tally & Benchmark & LCLS & $\frac{F O M_{L C L S}}{F O M_{\text {bench }}}$ \\
\hline Surface Current [n/source n] & $7.0 \mathrm{E}-4(1)$ & $6.2 \mathrm{E}-4(1)$ & 7 \\
\hline Matrix Cell Flux [n/cm²/source n] & $1.359 \mathrm{E}-4(9)$ & $1.292 \mathrm{E}-4(5)$ & - \\
\hline Stochastic Cell Flux [n/cm²/source n] & $5.02 \mathrm{E}-8(5)$ & $5.441 \mathrm{E}-8(7)$ & - \\
\hline 1 standard deviation uncerta & e last significan & lown in par & \\
\hline
\end{tabular}


Table II. Thermal neutron source uniformly distributed in matrix. Tally and FOM results for three sets of matrix material densities.

\begin{tabular}{|c|c|c|c|}
\hline \multicolumn{4}{|c|}{ Set1: $\rho 0=10[\mathrm{gm} / \mathrm{cc}], \rho 1=0.001[\mathrm{gm} / \mathrm{cc}]$} \\
\hline Tally & Benchmark & LCLS & $\frac{F O M_{L C L S}}{F O M_{\text {bench }}}$ \\
\hline Surface Current [n/source n] & $0.5417(4)$ & $0.5435(3)$ & 5.7 \\
\hline Matrix Cell Flux [n/cm²/source n] & $1.818 \mathrm{E}-4(2)$ & $1.824 \mathrm{E}-4(9)$ & - \\
\hline Stochastic Cell Flux [n/cm²/source n] & $6.046 \mathrm{E}-8(8)$ & $6.027 \mathrm{E}-8(7)$ & - \\
\hline \multicolumn{4}{|c|}{ Set 2: $\rho 0=10[\mathrm{gm} / \mathrm{cc}], \rho 1=0.1[\mathrm{gm} / \mathrm{cc}]$} \\
\hline Tally & Benchmark & LCLS & $\frac{F O M_{L C L S}}{F O M_{\text {bench }}}$ \\
\hline Surface Current [n/source n] & $0.4538(4)$ & $0.4531(4)$ & 4.8 \\
\hline Matrix Cell Flux [n/cm $/$ source n] & $1.801 \mathrm{E}-4(2)$ & $1.798 \mathrm{E}-4(1)$ & - \\
\hline Stochastic Cell Flux [n/cm²/source n] & $6.116 \mathrm{E}-8(8)$ & $6.127 \mathrm{E}-8(8)$ & - \\
\hline \multicolumn{4}{|c|}{ Set 3: $\rho 0=10[\mathrm{gm} / \mathrm{cc}], \rho 1=1[\mathrm{gm} / \mathrm{cc}]$} \\
\hline Tally & Benchmark & LCLS & $\frac{F O M_{L C L S}}{F O M_{\text {bench }}}$ \\
\hline Surface Current [n/source n] & $0.1765(3)$ & $0.1725(2)$ & 8.07 \\
\hline Matrix Cell Flux [n/cm $/$ source n] & $1.1256 \mathrm{E}-4(6)$ & $1.0969 \mathrm{E}-4(6)$ & - \\
\hline Stochastic Cell Flux [n/cm $/ \mathrm{cm}^{2} /$ source $\left.\mathrm{n}\right]$ & $3.938 \mathrm{E}-8(6)$ & $4.200 \mathrm{E}-8(6)$ & - \\
\hline
\end{tabular}

\section{CONCLUSIONS}

The cases examined test the effectiveness of the LCLS method for treating particle transport through a specified binary stochastic mixture. The stochastic spheres are modeled as black absorbers, and the effects of increased scattering in the matrix material are examined. The three matrix densities correspond to optical thicknesses of roughly $0.01,1$, and 10.

For both source distributions, LCLS and benchmark results compare closely for the first two matrix densities. As scattering in the matrix increases, LCLS accuracy degrades. The assumption of an exponential chord length distribution and the value chosen for the mean chord length may contribute to the loss in accuracy because sampling from equation 1 is performed more and more frequently as scattering increases. However, it is believed that the loss of accuracy is due principally to the assumption of Markovian transport made by the LCLS method. Markovian transport implies that a particle's future is independent of its past. In a homogenous medium, transport is Markovian. However, particle transport through a stochastic mixture is not Markovian because the distances to the surrounding material interfaces depend on the past trajectory of the particle. In the LCLS method, the sampled location of a stochastic sphere is discarded after a particle exits the stochastic sphere. Upon a scattering event in the matrix, a particle could intersect a sphere where there had previously been none. For this reason, as scattering in the matrix material increases the LCLS method is expected to overpredict the flux in the stochastic spheres. 
One of the interesting findings of this work lies in the optimization of the FOM for an ensemble averaged Monte Carlo result. The combination of geometric and stochastic randomness in the averaged benchmark result allows for the optimization of the number of particles run per realization. Because of the overhead cost to build a complex random cell geometry, the benchmark method is least efficient when the optimized run strategy requires many realizations with a relatively small number of particles per realization. Conversely, if the variance in the ensemble averaged result is not strongly affected by the randomness of the geometry, then an accurate result can be computed with only a few realizations, each running a large number of particles.

The point source case with the lowest matrix density shows a FOM ratio of 141 in favor of LCLS. The benchmark for this case required a large number of realizations with relatively few particles per realization. In addition, because the matrix is so transparent, the transport time per particle for both LCLS and benchmark is low. Therefore for the benchmark the total time is dominated by the time required to build all of the realizations. This is the reason that the FOM ratio is the highest for this case.

For the uniform matrix source and the lowest matrix density, the FOM ratio drops to 5.7. The geometric variability is greatly reduced by distributing the source through the matrix. This causes the efficiency of the benchmark to increase. The advantage in the LCLS method in this case is driven by the reduced transport time per particle for LCLS. Transport time per particle for LCLS is less because of the reduced number of cells and the simpler matrix cell as compared with the benchmark model. The geometric simplicity of LCLS is obtained at the expense of the additional chord length sampling and associated calculations.

\section{REFERENCES}

1. G.B. Zimmerman, M.L. Adams, "Algorithms for Monte Carlo Particle Transport in Binary Statistical Mixtures", Transactions of the American Nuclear Society, 64, pp. 286-294, (1991).

2. T.J. Donovan, Y. Danon, "Application of Monte Carlo Chord-Length Sampling Algorithms to Transport Through a Two-Dimensional Binary Stochastic Mixture", Nuclear Science \& Engineering, 143, pp. 1-14, (2003).

3. J.F. Briesmeister, editor, "MCNP'M - A General Monte Carlo N-Particle Transport Code", LA-13709-M, April 2000.

4. J.R. Johnson, J.R. Lebenhaft, M.J. Driscoll, "Burnup Reactivity and Isotopics of an HTGR Fuel Pebble", Transactions of the American Nuclear Society, 51 pp. 273-274, (2001).

5. S. Torquato, "Random Heterogeneous Materials: Microstructure and Macroscopic Properties", Springer-Verlag New York, (2002).

6. B. Su, G.C. Pomraning, "A Stochastic Description of a Broken Cloud Field", Transactions of the American Nuclear Society, 51, pp. 1969-1977, (1994). 